\title{
On the Uniqueness of the Fock Quantization of the Dirac Field in the Closed FRW Cosmology
}

\author{
Jerónimo Cortez, ${ }^{1}$ Ilda Inácio Rodrigues, ${ }^{2}$ \\ Mercedes Martín-Benito, ${ }^{3,4}$ and José Velhinho $\mathbb{1}^{2}$ \\ ${ }^{1}$ Departamento de Física, Facultad de Ciencias, Universidad Nacional Autónoma de México, 04510 Ciudad de México, Mexico \\ ${ }^{2}$ Faculdade de Ciências, Universidade da Beira Interior, R. Marquês d’Ávila e Bolama, 6201-001 Covilhã, Portugal \\ ${ }^{3}$ Instituto de Astrofísica e Ciências do Espaço, Faculdade de Ciências, Universidade de Lisboa, Ed. C8, Campo Grande, \\ 1749-016 Lisboa, Portugal \\ ${ }^{4}$ Departamento de Física Teórica, Universidad Complutense de Madrid, 28040 Madrid, Spain
}

Correspondence should be addressed to José Velhinho; jvelhi@ubi.pt

Received 27 November 2017; Revised 29 January 2018; Accepted 14 February 2018; Published 19 March 2018

Academic Editor: Claudio Dappiaggi

Copyright (C) 2018 Jerónimo Cortez et al. This is an open access article distributed under the Creative Commons Attribution License, which permits unrestricted use, distribution, and reproduction in any medium, provided the original work is properly cited.

The Fock quantization of free fields propagating in cosmological backgrounds is in general not unambiguously defined due to the nonstationarity of the space-time. For the case of a scalar field in cosmological scenarios, it is known that the criterion of unitary implementation of the dynamics serves to remove the ambiguity in the choice of Fock representation (up to unitary equivalence). Here, applying the same type of arguments and methods previously used for the scalar field case, we discuss the issue of the uniqueness of the Fock quantization of the Dirac field in the closed FRW space-time proposed by D'Eath and Halliwell.

\section{Introduction}

The physics of the very early universe, and in particular relevant quantum phenomena, can nowadays be tested, comparing the predictions of theoretical models against quite accurate observational data. Besides scalar fields, it is therefore important to explore the impact of different matter sources as well (in this respect, see [1]). Concerning fermion fields, and in particular Dirac spinors, a consistent framework to deal with the corresponding quantum field theory in a cosmological context was put forward several years ago by D'Eath and Halliwell [2] (see also [3] for a more general discussion). In particular, [2] considers a concrete Fock quantization of the (fully inhomogeneous) Dirac field, on homogeneous and isotropic background space-time, namely, the closed Friedmann-Robertson-Walker (FRW) cosmological model.

As it is typically the case regarding the quantization of systems with an infinite number of degrees of freedom, also in the quantization of the Dirac field in curved space-time, one must face the issue of ambiguity, or lack of uniqueness in the quantization procedure. Just like in the scalar field case, the ambiguity in the quantization can be seen to lie in the choice of a so-called complex structure in the space of solutions (of the Dirac equation in this case). For the analogous problem concerning the scalar field, it was shown [4-8] that the requirement of a unitary implementation of the dynamics, combined with a natural implementation of spatial symmetries, leads to a unique quantization (modulo unitary equivalence), thus removing the ambiguity problem. The unitary evolution criterion replaces here the well-known requirements based on invariance under space-time symmetries (such as the Poincaré group or the symmetries of the de Sitter space) or on stationarity, which are unavailable, for example, in typical inflationary and cosmological scenarios.

In the previous work [9], it was shown that D'Eath and Halliwell's [2] quantization of the Dirac field in the closed FRW cosmology satisfies requirements similar to those just mentioned in the scalar field context; namely, the complex structure chosen in [2] is invariant under the symmetries of the field equations (which of course include $S O(4)$, the isometry group of the spatial sections) and admits a unitary implementation of the dynamics. Most importantly, it was 
shown that a large class of seemingly natural alternatives to D'Eath and Halliwell's quantization actually lead to the same quantization (modulo unitary equivalence) once unitary implementation of the dynamics is required, thus providing a strong indication that full uniqueness of the quantization result is valid for the Dirac field, in perfect correspondence with the previous results obtained for the scalar field case. Moreover, such a uniqueness result was indeed obtained later on, as a corollary of a more general analysis presented in [10] (see also [11, 12]). However, the approach taken in [10] was substantially different from the line of reasoning previously adopted for the scalar field. In particular, in [10], two different types of ambiguities were addressed simultaneously. In fact, besides the usual ambiguity in the choice of the quantum representation for a fixed classical field description, time-dependent scalings of the fermion field modes (after an appropriate mode decomposition) were also allowed, effectively modifying the original D'Eath and Halliwell parametrization of the system. In this respect, a sort of time-dependent complex structure was introduced in [10], leading to a more effective and economic mathematical treatment. On the other hand, this different perspective might have contributed to a less than full appreciation of the results obtained in [10]. In the current work, we recover the line of reasoning followed in the scalar field case, presenting a new and fully independent proof of part of the results of [10]. In particular, we will show that, preserving D'Eath and Halliwell's field description, the quantization of the Dirac field put forward in [2] is essentially unique, in the sense that all Fock quantizations determined by SO(4)-invariant complex structures admitting a unitary implementation of the dynamics are unitarily equivalent.

Let us mention that there are similarities between the unitary evolution requirement and the so-called Hadamard condition, in the sense that some type of ultraviolet regularity is imposed in both cases. Moreover, for space-time with compact spatial sections, all (pure Fock) Hadamard states lead to unitarily equivalent quantizations $[13,14]$. We present here a different approach towards uniqueness of the quantization, insisting on a unitary implementation of the dynamics at the quantum level. A brief discussion concerning the relation between these two approaches is presented in Section 5.

\section{The Model and Its Quantization}

In this section, we present a very brief review of the quantum treatment of the Dirac field in the unit three-sphere $S^{3}$, following $[2,9]$.

Let us consider the closed FRW cosmological model, with metric

$$
d s^{2}=e^{2 \alpha(\eta)}\left(-d \eta^{2}+d \Omega_{3}^{2}\right),
$$

where $\eta$ denotes conformal time and $d \Omega_{3}^{2}$ is the metric on $S^{3}$. The massive Dirac spinor $\Psi$ is taken in the Weyl representation; that is, it is described by a pair of two-component spinors, $\phi^{A}$ and $\bar{\chi}_{A^{\prime}}$, with opposite chirality. The spinors $\phi_{A}$ and $\bar{\chi}_{A^{\prime}}$ can be expanded in complete bases of spinor harmonics on $S^{3}$ provided by the eigenmodes of the Dirac operator, hereby denoted by $\rho_{A}^{n p}, \bar{\sigma}_{A}^{n p}$ and $\bar{\rho}_{A^{\prime}}^{n p}, \sigma_{A^{\prime}}^{n p}$. Here, $n \epsilon$ $\mathbb{N}$, the degeneracy of each eigenspace is

$$
g_{n}=(n+1)(n+2)=\omega_{n}^{2}-\frac{1}{4},
$$

with $\omega_{n}=n+3 / 2$, and the label $p=1, \ldots, g_{n}$ accounts for the degeneracy of the eigenspaces.

Explicitly, the two-component spinors (and their Hermitian conjugates) can be expanded in modes as follows:

$$
\begin{aligned}
& \phi_{A}(x) \\
& =\frac{e^{-3 \alpha(\eta) / 2}}{2 \pi} \sum_{n p q} \breve{\alpha}_{n}^{p q}\left[m_{n p}(\eta) \rho_{A}^{n q}(\mathbf{x})+\bar{r}_{n p}(\eta) \bar{\sigma}_{A}^{n q}(\mathbf{x})\right], \\
& \bar{\chi}_{A^{\prime}}(x) \\
& =\frac{e^{-3 \alpha(\eta) / 2}}{2 \pi} \sum_{n p q} \breve{\beta}_{n}^{p q}\left[\bar{s}_{n p}(\eta) \bar{\rho}_{A^{\prime}}^{n q}(\mathbf{x})+t_{n p}(\eta) \sigma_{A^{\prime}}^{n q}(\mathbf{x})\right],
\end{aligned}
$$

with

$$
\sum_{n p q}:=\sum_{n=0}^{\infty} \sum_{p=1}^{g_{n}} \sum_{q=1}^{g_{n}} .
$$

The Grassmann variables $m_{n p}, r_{n p}, s_{n p}$, and $t_{n p}$ therefore describe the fermionic degrees of freedom, after mode decomposition. In the above expressions, the coefficients $\breve{\alpha}_{n}^{p q}$ and $\breve{\beta}_{n}^{p q}$ are the matrix elements of two constant matrices $\breve{\alpha}_{n}$ and $\breve{\beta}_{n}$, each of dimension $g_{n}$ and block-diagonal form, with blocks given, respectively, by

$$
\begin{aligned}
& \left(\begin{array}{cc}
1 & 1 \\
1 & -1
\end{array}\right), \\
& \left(\begin{array}{cc}
1 & -1 \\
-1 & -1
\end{array}\right) .
\end{aligned}
$$

They are included by convenience, to avoid dynamical couplings between mode components with different values of $p$.

The mode components of the Dirac equation, deduced from the Einstein-Dirac action, can be summarized in the following set of differential equations (and corresponding complex conjugates) [2]:

$$
\begin{aligned}
& x_{n p}^{\prime}=i \omega_{n} x_{n p}-i m e^{\alpha} \bar{y}_{n p}, \\
& y_{n p}^{\prime}=i \omega_{n} y_{n p}+i m e^{\alpha} \bar{x}_{n p} .
\end{aligned}
$$

In the above equations, $m$ stands for the mass of the fermion field, and the prime denotes derivative with respect to conformal time. We are adopting here the notation $\left(x_{n p}, y_{n p}\right)$ to denote any of the sets of pairs $\left(m_{n p}, s_{n p}\right)$ or $\left(t_{n p}, r_{n p}\right)$.

As mentioned in the Introduction, the ambiguity in the Fock quantization of the Dirac field resides in the choice of a complex structure in the space of solutions of the Dirac equation. This in turn amounts to a choice of a set of classical 
annihilation- and creation-like variables, to be quantized as annihilation and creation operators (see [9] for details).

The preferred choice of annihilation- and creation-like variables considered in [2] diagonalizes the Hamiltonian and is defined as follows. The annihilation-like variables of the particles and antiparticles are chosen to be

$$
\begin{aligned}
& a_{n p}^{(x, y)}=\sqrt{\frac{1}{2}-\frac{1}{2 \xi_{n}}} x_{n p}+\sqrt{\frac{1}{2}+\frac{1}{2 \xi_{n}}} \bar{y}_{n p}, \\
& b_{n p}^{(x, y)}=\sqrt{\frac{1}{2}+\frac{1}{2 \xi_{n}}} \bar{x}_{n p}-\sqrt{\frac{1}{2}-\frac{1}{2 \xi_{n}}} y_{n p},
\end{aligned}
$$

with

$$
\xi_{n}=\sqrt{1+\left(\frac{m e^{\alpha}}{\omega_{n}}\right)^{2}}
$$

The creation-like variables $a_{n p}^{(x, y) \dagger}:=\bar{a}_{n p}^{(x, y)}$ and $b_{n p}^{(x, y) \dagger}:=$ $\bar{b}_{n p}^{(x, y)}$ are their complex conjugates. When a complete Hamiltonian analysis of the Einstein-Dirac action is performed, one finds that these variables indeed satisfy the Dirac brackets characteristic of annihilation- and creation-like variables for particles and antiparticles; namely,

$$
\begin{aligned}
\left\{a_{n p}^{(x, y)}, a_{n p}^{(x, y) \dagger}\right\}=\left\{b_{n p}^{(x, y)}, b_{n p}^{(x, y) \dagger}\right\}=- & \\
& \left\{a_{n p}^{(x, y)}, b_{n p}^{(x, y)}\right\}=0 .
\end{aligned}
$$

Let us call reference complex structure and reference quantization the ones determined by the choice of variables introduced above.

The main feature of this quantization is that it allows a unitary implementation of the classical field dynamics in the quantum theory. Let us see exactly what this means. Since both the equations of motion and the relations (7) are linear, it is clear that the variables defined by relations (7) evolve linearly in time. In particular, the evolution from an arbitrary initial time $\eta_{0}$ to any other time $\eta$ is given by a Bogoliubov transformation of the form

$$
\left(\begin{array}{c}
a_{n p}^{(x, y)} \\
b_{n p}^{(x, y) \dagger}
\end{array}\right)_{\eta}=B_{n}\left(\eta, \eta_{0}\right)\left(\begin{array}{c}
a_{n p}^{(x, y)} \\
b_{n p}^{(x, y) \dagger}
\end{array}\right)_{\eta_{0}},
$$

with

$$
B_{n}=\left(\begin{array}{cc}
\alpha_{n}^{f} & \beta_{n}^{f} \\
\beta_{n}^{g} & \alpha_{n}^{g}
\end{array}\right),
$$

where $\alpha_{n}^{f}$ and $\beta_{n}^{f}$ are coefficients (dependent on $\eta$ and $\eta_{0}$ ) whose full expression can be found in [9].

It turns out that a classical transformation of type (10) can be unitarily implemented (in the Fock representation defined by the complex structure corresponding to the choice of variables (7)) if and only if [15] the sum

$$
\sum_{n} g_{n}\left(\left|\beta_{n}^{f}\right|^{2}+\left|\beta_{n}^{g}\right|^{2}\right)
$$

is finite. Of course, since in the present case one is interested in the unitary implementation of the dynamics, one must require that the above sum be finite for all values of time $\eta$ and $\eta_{0}$, which was proven to be the case [9].

\section{Alternative Complex Structures and Unitarity Condition}

The question that we now want to answer is the following: is the quantization described in the previous section unique? In what sense? We have learned from the analysis of similar cases involving scalar fields that the requirement of unitary implementation of the dynamics is a successful criterion in the selection of quantum representations. In fact, together with the requirement of invariance of the complex structure under remaining spatial symmetries, the unitary dynamics condition selects a unique quantization (modulo unitary equivalence) for the scalar field propagating in a nonstationary homogeneous and isotropic space-time background [7], typical of cosmological scenarios. Note that the invariance of the complex structure guarantees a natural unitary implementation of the symmetries in question, and it is therefore reasonable to restrict attention to those invariant complex structures. Of course, our considerations should be extended to noninvariant complex structures whenever the physical conditions require it or if physically relevant noninvariant complex structures are known to exist. That is not the case in the present situation, to the best of our knowledge. On the other hand, the unitary implementation of the dynamics cannot be made via complex structures that remain invariant under evolution, for the simple reason that such complex structures do not exist, due to the lack of stationarity.

As shown in detail in [10], the general form of a complex structure that remains invariant under the action of the isometry group of $S^{3}$ on the space of two-component spinors is very simple to characterize. Such an invariant complex structure is associated with a different choice of annihilation- and creation-like variables $\left\{\widetilde{a}_{n p}^{(x, y)}, \widetilde{b}_{n p}^{(x, y)}, \widetilde{a}_{n p}^{(x, y) \dagger}, \widetilde{b}_{n p}^{(x, y) \dagger}\right\}$, related to our reference variables (7) by means of a transformation (and its complex conjugate) of the type

$$
\left(\begin{array}{c}
\tilde{a}_{n p}^{(x, y)} \\
\tilde{b}_{n p}^{(x, y) \dagger}
\end{array}\right)=K_{n}\left(\begin{array}{c}
a_{n p}^{(x, y)} \\
b_{n p}^{(x, y) \dagger}
\end{array}\right),
$$

with

$$
K_{n}=\left(\begin{array}{cc}
\kappa_{n}^{f} & \lambda_{n}^{f} \\
\lambda_{n}^{g} & \kappa_{n}^{g}
\end{array}\right),
$$

where the time-independent matrices $K_{n}, n \in \mathbb{N}$, are arbitrary $2 \times 2$ unitary matrices; that is, they satisfy the relations

$$
\begin{gathered}
\left|\kappa_{n}^{f}\right|^{2}+\left|\lambda_{n}^{f}\right|^{2}=1, \\
\left|\kappa_{n}^{g}\right|^{2}+\left|\lambda_{n}^{g}\right|^{2}=1, \\
\kappa_{n}^{f} \bar{\lambda}_{n}^{g}+\lambda_{n}^{f} \bar{\kappa}_{n}^{g}=0,
\end{gathered}
$$

as follows from (9). 
Among the large class of quantum representations of the Dirac field determined by the invariant complex structures as above, there are certainly an infinite number of representations that are not unitarily equivalent to our reference quantization. In fact, the condition for unitary equivalence reads $[15,16]$

$$
\sum_{n} g_{n}\left(\left|\lambda_{n}^{f}\right|^{2}+\left|\lambda_{n}^{g}\right|^{2}\right)<\infty
$$

which is obviously not satisfied by an arbitrary sequence of matrices $K_{n}$.

At this point, it should be mentioned that there is an important difference-first noted in [9] - with respect to the previously studied scalar field case, which we will now address. Note that orthogonality conditions (15) (contrary to corresponding symplectic conditions in the scalar field case) can be fulfilled with $\lambda_{n}^{f}=\lambda_{n}^{g}=1, \kappa_{n}^{f}=\kappa_{n}^{g}=0$. It is clear that a transformation of this type simply interchanges particles with antiparticles and therefore has no physical meaning, since that distinction is conventional to begin with. However, if one allows such a transformation for an infinite number of modes, one ends up with two formally inequivalent quantizations, since the equivalence condition (16) is clearly violated. In order to eliminate the artificially inequivalent complex structures associated with these types of transformations, we will adhere to a fixed convention as to what is called particle and antiparticle, which we will not allow to change (except possibly for a finite number of modes). A concrete implementation of this notion is to require, for example, that the sequences $\left\{\kappa_{n}^{f}\right\}$ and $\left\{\kappa_{n}^{g}\right\}$ in (14) have no subsequences that tend to zero, which we will assume from now on. There still remains, of course, a large class of physically meaningful complex structures.

We are going to show, precisely, that once unitary implementation of the dynamics is imposed as a requirement, only those complex structures that satisfy (16) survive. As a preparation, let us state a preliminary consequence of the existence of unitary dynamics.

One can easily show (see [4]) that a complex structure associated with a choice of variables (13), determined by matrices $K_{n}$, allows a unitary implementation of the dynamics if and only if the time-dependent transformation determined by the sequence of matrices $K_{n} B_{n}\left(\eta, \eta_{0}\right) K_{n}^{-1}$ is unitarily implementable with respect to our reference quantization. A straightforward computation shows that, as a consequence, the following two sequences must be square-summable, $\forall \eta$ :

$$
\begin{aligned}
& \sqrt{g_{n}}\left(\left(\kappa_{n}^{f}\right)^{2} \beta_{n}^{f}-\left(\lambda_{n}^{f}\right)^{2} \beta_{n}^{g}+\kappa_{n}^{f} \lambda_{n}^{f}\left(\alpha_{n}^{g}-\alpha_{n}^{f}\right)\right), \\
& \sqrt{g_{n}}\left(\left(\kappa_{n}^{g}\right)^{2} \beta_{n}^{g}-\left(\lambda_{n}^{g}\right)^{2} \beta_{n}^{f}+\kappa_{n}^{g} \lambda_{n}^{g}\left(\alpha_{n}^{f}-\alpha_{n}^{g}\right)\right) .
\end{aligned}
$$

Since we know that (i) the sequences $\sqrt{g_{n}} \beta_{n}^{f}$ and $\sqrt{g_{n}} \beta_{n}^{g}$ are square-summable, because the sum (12) was shown to be finite [9], and (ii) the sequences $\kappa_{n}$ and $\lambda_{n}$ are bounded, it follows (from linearity of the set of square-summable sequences) that necessarily

$$
\begin{aligned}
& \sum_{n} g_{n}\left|\kappa_{n}^{f} \lambda_{n}^{f}\left(\alpha_{n}^{g}-\alpha_{n}^{f}\right)\right|^{2}<\infty, \\
& \sum_{n} g_{n}\left|\kappa_{n}^{g} \lambda_{n}^{g}\left(\alpha_{n}^{f}-\alpha_{n}^{g}\right)\right|^{2}<\infty .
\end{aligned}
$$

These conditions are therefore consequences of the requirement of unitarity of the dynamics. We will take these as the starting point for our next section, where we will prove that conditions (18) in fact imply that (16) is satisfied.

\section{Uniqueness of the Quantization}

A detailed asymptotic analysis of the evolution matrices $B_{n}$ (11) was performed in [9]. Following the same procedure that allowed obtaining an expression for $\beta_{n}^{h}$ in the limit of large $n$ (equation (3.18) in [9]), we obtain the following expression for $\alpha_{n}^{g}-\alpha_{n}^{f}, \forall \eta$, in the limit of large $n$ :

$$
\begin{aligned}
& \left(\alpha_{n}^{g}-\alpha_{n}^{f}\right)\left(\eta, \eta_{0}\right)=2 i\left\{\left[f_{2}^{n}(\eta) f_{2}^{n}\left(\eta_{0}\right)-f_{1}^{n}(\eta)\right.\right. \\
& \left.\cdot f_{1}^{n}\left(\eta_{0}\right)\right] e^{-\Im\left(\Lambda_{n}^{1}(\eta)\right)} \sin \left(\omega_{n} \Delta \eta\right. \\
& \left.+\int_{\eta_{0}}^{\eta} \mathfrak{R}\left(\Lambda_{n}^{1}(\bar{\eta})\right) d \bar{\eta}\right)+\left[f_{1}^{n}(\eta) f_{2}^{n}\left(\eta_{0}\right)+f_{2}^{n}(\eta)\right. \\
& \left.\cdot f_{1}^{n}\left(\eta_{0}\right)\right]\left|\Gamma_{n}\right| e^{\Delta \alpha}\left[e ^ { - \Im ( \Lambda _ { n } ^ { 1 } ( \eta ) ) } \operatorname { s i n } \left(\omega_{n} \Delta \eta\right.\right. \\
& \left.+\int_{\eta_{0}}^{\eta} \mathfrak{R}\left(\Lambda_{n}^{1}(\bar{\eta})\right) d \bar{\eta}+\varphi_{n}\right)-e^{\Im\left(\Lambda_{n}^{2}(\eta)\right)} \sin \left(\omega_{n} \Delta \eta\right. \\
& \left.\left.\left.+\int_{\eta_{0}}^{\eta} \mathfrak{R}\left(\Lambda_{n}^{2}(\bar{\eta})\right) d \bar{\eta}-\varphi_{n}\right)\right]\right\} .
\end{aligned}
$$

In the above expression, we have $\Delta \eta=\eta-\eta_{0}, \Delta \alpha=\alpha(\eta)-$ $\alpha\left(\eta_{0}\right)$,

$$
\begin{aligned}
& f_{1}^{n}=\sqrt{\frac{1}{2}-\frac{1}{2 \xi_{n}}}=\frac{m e^{\alpha}}{2 \omega_{n}}+\mathcal{O}\left(\omega_{n}^{-2}\right), \\
& f_{2}^{n}=\sqrt{1-\left|f_{1}^{n}\right|^{2}}=1+\mathcal{O}\left(\omega_{n}^{-2}\right), \\
& \Gamma_{n}=\frac{m e^{\alpha\left(\eta_{0}\right)}}{2 \omega_{n}+i \alpha^{\prime}\left(\eta_{0}\right)}=\frac{m e^{\alpha\left(\eta_{0}\right)}}{2 \omega_{n}}+\mathcal{O}\left(\omega_{n}^{-2}\right),
\end{aligned}
$$

$\varphi_{n}$ is the phase of $\Gamma_{n}$, and $\Lambda_{n}^{1}$ and $\Lambda_{n}^{2}$ are time-dependent functions, defined in the appendix of [9], which have the property of being of order $\mathcal{O}\left(\omega_{n}^{-1}\right)$ in the limit of large $n$.

Taking into account the asymptotic limits of the coefficients $f_{1}^{n}, \Gamma_{n}, \Lambda_{n}^{1}$, and $\Lambda_{n}^{2}$, it follows that all the terms in 
$\sqrt{g_{n}} \kappa_{n}^{f} \lambda_{n}^{f}\left(\alpha_{n}^{g}-\alpha_{n}^{f}\right)$ are square-summable, with the possible exception of the term

$$
\begin{aligned}
& 2 i \sqrt{g_{n}} \kappa_{n}^{f} \lambda_{n}^{f} f_{2}^{n}(\eta) f_{2}^{n}\left(\eta_{0}\right) e^{-\Im\left(\Lambda_{n}^{1}\right)} \\
& \quad \cdot \sin \left(\omega_{n} \Delta \eta+\int_{\eta_{0}}^{\eta} \Re\left(\Lambda_{n}^{1}(\bar{\eta})\right) d \bar{\eta}\right) .
\end{aligned}
$$

However, since we are assuming that the sequence $\sqrt{g_{n}} \kappa_{n}^{f} \lambda_{n}^{f}\left(\alpha_{n}^{g}-\alpha_{n}^{f}\right)$ itself is square-summable, it follows that sequence $(21)$ is square-summable as well. Moreover, since $f_{2}^{n}(\eta) f_{2}^{n}\left(\eta_{0}\right) e^{-\Im\left(\Lambda_{n}^{1}\right)}$ actually tends to one in the large $n$ limit, we conclude that

$$
\sum_{n} g_{n}\left|\kappa_{n}^{f} \lambda_{n}^{f}\right|^{2} \sin ^{2}\left(\omega_{n} \Delta \eta+\int_{\eta_{0}}^{\eta} \Re\left(\Lambda_{n}^{1}(\bar{\eta})\right) d \bar{\eta}\right)<\infty .
$$

Obviously, the same reasoning leads to the conclusion that

$$
\sum_{n} g_{n}\left|\kappa_{n}^{g} \lambda_{n}^{g}\right|^{2} \sin ^{2}\left(\omega_{n} \Delta \eta+\int_{\eta_{0}}^{\eta} \Re\left(\Lambda_{n}^{1}(\bar{\eta})\right) d \bar{\eta}\right)<\infty .
$$

These two conditions are therefore consequences of (18), which are in turn a consequence of our requirement of unitary implementation of the dynamics. From this point on, the proof that (22)-(23) implies (16) can be done along the lines of the argument presented in [6]. Let us then introduce the shifted time $T:=\eta-\eta_{0}$ and rewrite the elements of the sequence appearing in (22) in the form

$$
\sqrt{g_{n}} \kappa_{n}^{f} \lambda_{n}^{f} \sin \left[\omega_{n} T+\int_{0}^{T} d \bar{\eta} \Re\left(\Lambda_{n}^{1}\left(\bar{\eta}+\eta_{0}\right)\right)\right] .
$$

The function

$$
\begin{aligned}
z(T):= & \lim _{M \rightarrow \infty} \sum_{n=1}^{M} g_{n}\left|\kappa_{n}^{f}\right|^{2}\left|\lambda_{n}^{f}\right|^{2} \\
& \cdot \sin ^{2}\left[\omega_{n} T+\int_{0}^{T} d \bar{\eta} \mathfrak{R}\left(\Lambda_{n}^{1}\left(\bar{\eta}+\eta_{0}\right)\right)\right]
\end{aligned}
$$

therefore exists for all $T$ in the domain of $\eta-\eta_{0}$. In particular, $z(T)$ is well defined on some closed subinterval $\overline{\mathbb{}}_{L}=[a, a+L]$, where $L$ is some finite positive number.

Luzin's theorem [17] guarantees that, for every $\delta>0$, there exist (i) a measurable set $E_{\delta} \subset \overline{\mathbb{D}}_{L}$ such that its complement $\bar{E}_{\delta}$ with respect to $\bar{\square}_{L}$ satisfies $\int_{\bar{E}_{\delta}} d T<\delta$ and (ii) a function $F_{\delta}(T)$ which is continuous on $\overline{\mathbb{Q}}_{L}$ and coincides with $z(T)$ in $E_{\delta}$. In particular, defining $I_{\delta}:=\int_{E_{\delta}} F_{\delta}(T) d T$, we obtain from Luzin's theorem

$$
\begin{aligned}
& \sum_{n=1}^{M} g_{n}\left|\kappa_{n}^{f}\right|^{2}\left|\lambda_{n}^{f}\right|^{2} \\
& \quad \cdot \int_{E_{\delta}} \sin ^{2}\left[\omega_{n} T+\int_{0}^{T} d \bar{\eta} \mathfrak{R}\left(\Lambda_{n}^{1}\left(\bar{\eta}+\eta_{0}\right)\right)\right] d T \\
& \quad \leq \int_{E_{\delta}} z(T) d T=I_{\delta}, \quad \forall M \in \mathbb{N}^{+} .
\end{aligned}
$$

This inequality provides us with a bound on $\sum g_{n}\left|\kappa_{n}^{f}\right|^{2}\left|\lambda_{n}^{f}\right|^{2}$. To show it, we note that, $\forall n$,

$$
\begin{aligned}
\int_{E_{\delta}} & \sin ^{2}\left[\omega_{n} T+\int_{0}^{T} d \bar{\eta} \Re\left(\Lambda_{n}^{1}\left(\bar{\eta}+\eta_{0}\right)\right)\right] d T \\
= & \int_{\overline{\mathbb{Q}}_{L}} \sin ^{2}\left[\omega_{n} T+\int_{0}^{T} d \bar{\eta} \Re\left(\Lambda_{n}^{1}\left(\bar{\eta}+\eta_{0}\right)\right)\right] d T \\
& -\int_{\bar{E}_{\delta}} \sin ^{2}\left[\omega_{n} T+\int_{0}^{T} d \bar{\eta} \Re\left(\Lambda_{n}^{1}\left(\bar{\eta}+\eta_{0}\right)\right)\right] d T \\
\geq & \int_{\overline{\mathbb{D}}_{L}} \sin ^{2}\left[\omega_{n} T+\int_{0}^{T} d \bar{\eta} \Re\left(\Lambda_{n}^{1}\left(\bar{\eta}+\eta_{0}\right)\right)\right] d T-\delta .
\end{aligned}
$$

Applying now an integration by parts and a bound just like in the appendix of [6], we find the following for the integral over $\overline{\bar{D}}_{L}$ :

$$
\begin{aligned}
& \int_{\overline{\mathbb{D}}_{L}} \sin ^{2}\left[\omega_{n} T+\int_{0}^{T} d \bar{\eta} \Re\left(\Lambda_{n}^{1}\left(\bar{\eta}+\eta_{0}\right)\right)\right] d T \\
& \geq \frac{L}{2}-\frac{1}{2 \omega_{n_{0}}(1-D)}-\frac{\int_{\overline{\mathbb{D}}_{L}}\left|C^{\prime}\left(T+\eta_{0}\right)\right| d T}{4 \omega_{n_{0}}^{3}(1-D)^{2}} \\
& :=\Lambda_{n_{0}} .
\end{aligned}
$$

Here, $C$ is the coefficient of the term in $\omega_{n}^{-1}$ of the Laurent series expansion of $\Re\left(\Lambda_{n}^{1}\right)$. This expression is valid for $n \geq n_{0}$, where $n_{0}$ is any fixed (positive) integer such that $\omega_{n_{0}}^{2}$ is larger than the maximum of the function $\left|C\left(T+\eta_{0}\right)\right| /(2 D)$ in the interval $\bar{\rrbracket}_{L}$, and $D<1$ is any fixed constant. We are assuming also that $n_{0}$ is such that $\Lambda_{n_{0}}>0$ (which can certainly be achieved with an appropriate choice, since $\Lambda_{n_{0}}$ tends to $L / 2$ when $n_{0}$ tends to infinity).

Let us introduce the above result in the last inequality of (27), to obtain

$$
\begin{aligned}
& \int_{E_{\delta}} \sin ^{2}\left[\omega_{n} T+\int_{0}^{T} d \bar{\eta} \mathfrak{R}\left(\Lambda_{n}^{1}\left(\bar{\eta}+\eta_{0}\right)\right)\right] d T \\
& \quad \geq \Lambda_{n_{0}}-\delta
\end{aligned}
$$

We choose now $\delta$ such that $\Lambda_{n_{0}}>\delta$, which is certainly possible. Then, it follows from (26) that, for all $M \geq n_{0}$,

$$
\sum_{n=n_{0}}^{M} g_{n}\left|\kappa_{n}^{f}\right|^{2}\left|\lambda_{n}^{f}\right|^{2} \leq \frac{I_{\delta}}{\Lambda_{n_{0}}-\delta}
$$

Since $n_{0}$ is fixed and the above bound is true for arbitrarily large $M$, it follows that the sequence $\sqrt{g_{n}} \kappa_{n}^{f} \lambda_{n}^{f}$ is squaresummable.

At this point, let us recall from the discussion in the previous section that, without loss of physical generality, the sequence $\left\{\kappa_{n}^{f}\right\}$ can be assumed to be bounded from below. Thus, it follows moreover that the sequence $\sqrt{g_{n}} \lambda_{n}^{f}$ is square-summable. Finally, since obviously the same reasoning applies to (23), one is led to the conclusion that condition 
(16) is satisfied. It is therefore proven that the quantization of the Dirac field put forward in [2] is indeed unique up to unitary equivalence, under the requirements of invariance of the complex structure and unitary implementation of the dynamics.

\section{Conclusions and Discussion}

We have analyzed the issue of the uniqueness of the Fock quantization of the Dirac field in the closed FRW spacetime proposed by D'Eath and Halliwell [2]. We have worked with a fixed parametrization of the system, leaving aside the extra freedom that resides in the possibility of applying (to the variables to be quantized) further time-dependent transformations. It was therefore possible to address the uniqueness issue in exactly the same way as previously done for the scalar field case. In this sense, the results obtained here are more restricted than those of [10] but were proven in a fully independent way, using arguments that are more familiar to the quantum cosmology literature. It was fully confirmed that once a convention for particles-antiparticles is agreed upon, D'Eath and Halliwell's Fock quantization is indeed unique, subjected to the requirements of invariance of the complex structure under the group of spatial isometries and unitary implementation of the dynamics in Fock space.

A question that deserves discussion is the following. It has been argued by several authors that the so-called Hadamard states and corresponding quantum representations are physically privileged for quantum field theory in curved spacetime since, in particular, they allow a regularization of the stress-energy tensor and a well-defined (perturbative) construction of interacting theories. Moreover, it is known-as a consequence of more general results in [14] and following previous results for the scalar field - that, for the Dirac field in globally hyperbolic space-time with compact spatial sections, all pure Fock Hadamard states lead to unitarily equivalent quantizations. In the special case of cosmological space-time, such as the case considered in the present paper, another privileged family of states is known, that of adiabatic states, and again all adiabatic states (of sufficiently high order) give rise to unitarily equivalent representations (in the spatially compact case) [18]. Moreover, adiabatic states (of sufficiently high order) and Hadamard states belong to the same unitary equivalence class (in the spatially compact case). Thus, criteria leading to unitarily equivalent quantizations (in the spatially compact case) do exist, and in that strict sense the results of the current paper bring essentially no novelty, except that of an alternative approach.

A similar question emerged in the previous studies of analogous (e.g., using the criteria of unitary implementation of the dynamics) uniqueness of quantization results concerning the scalar field [4-8]. It is again well known that, for the scalar field in globally hyperbolic space-time (with compact spatial sections), all pure Fock Hadamard states give rise to unitarily equivalent quantizations [13]. For the case of the scalar field in the closed FRW space-time, the relation between the quantum representation selected by the unitary evolution requirement and the one provided by Hadamard states was discussed in some detail in [7], with the following conclusions. Once the time-dependent scaling $\phi \rightarrow \varphi=a \phi$ is taken into account, where $a$ is the FRW scale factor, the two criteria lead to equivalent quantizations. In more precise terms, the unique (unitary equivalence class of) quantum representation determined by the requirement of unitary implementation of the classical dynamics of the field $\varphi$ induces a Fock quantization of the original field $\phi$ which is unitarily equivalent to the ones associated with both adiabatic and Hadamard states. (See [19] for a general definition of adiabatic states and $[20,21]$ for the special FRW setting. In the particular case of compact spatial sections, it can be shown that all adiabatic and Hadamard states give rise to unitarily equivalent representations [21].)

If one believes, as we do, that preserving unitarity of the dynamics as much as possible is a desirable aspect in quantum physics, the fact that this perspective actually leads to a quantum theory that is equivalent to the one associated with the celebrated Hadamard states appears by itself as an interesting and reassuring result.

Based on the previous experience with the scalar field, we likewise expect that, in the current context of the Dirac field in the closed FRW space-time, both Hadamard states and the requirement of unitary implementation of the dynamics would lead to essentially equivalent quantizations. However, the detailed analysis of the relation between the two different approaches in the Dirac field case seems rather involved, in comparison with the previous study concerning the scalar field [7], and it falls outside the scope of the present work.

\section{Conflicts of Interest}

The authors declare that there are no conflicts of interest regarding the publication of this paper.

\section{Acknowledgments}

The authors are immensely grateful to B. Elizaga Navascués and G. A. Mena Marugán for their help, insight, and constant support. This work was partially supported by the Research Grants MICINN/MINECO Project no. FIS2014-54800-C2-2$P$ from Spain and DGAPA-UNAM IN113115 and CONACyT 237351 from Mexico. José Velhinho would like to acknowledge the COST Action CA16104 GWverse, supported by COST (European Cooperation in Science and Technology). In addition, Mercedes Martín-Benito would like to acknowledge the financial support from the Portuguese Foundation for Science and Technology (FCT, Grant no. IF/00431/2015).

\section{References}

[1] B. Elizaga Navascués, G. A. Mena Marugán, and M. MartínBenito, "Fermions in hybrid loop quantum cosmology," Physical Review D: Particles, Fields, Gravitation and Cosmology, vol. 96, no. 4, Article ID 046005, 2017.

[2] P. D. D'Eath and J. J. Halliwell, "Fermions in quantum cosmology," Physical Review D: Particles, Fields, Gravitation and Cosmology, vol. 35, no. 4, pp. 1100-1123, 1987.

[3] J. Dimock, "Dirac quantum fields on a manifold," Transactions of the American Mathematical Society, vol. 269, no. 1, pp. 133-147, 1982. 
[4] A. Corichi, J. Cortez, G. A. Marugán, and J. M. Velhinho, "Quantum Gowdy T $\mathrm{T}^{3}$ model: a uniqueness result," Classical and Quantum Gravity, vol. 23, no. 22, pp. 6301-6319, 2006.

[5] G. J. F. Barbero, D. G. Vergel, and E. J. S. Villasẽor, "Quantum unitary evolution of linearly polarized $S^{1} \times S^{2}$ and $S^{3}$ Gowdy models coupled to massless scalar fields," Classical and Quantum Gravity, vol. 25, no. 8, Article ID 085002, 2008.

[6] J. Cortez, G. A. Mena Marugán, and J. M. Velhinho, "Fock quantization of a scalar field with time dependent mass on the three-sphere: Unitarity and uniqueness," Physical Review D: Particles, Fields, Gravitation and Cosmology, vol. 81, no. 4, Article ID 044037, 2010.

[7] J. Cortez, G. A. Mena Marugán, J. Olmedo, and J. M. Velhinho, "Criteria for the determination of time dependent scalings in the Fock quantization of scalar fields with a time dependent mass in ultrastatic spacetimes," Physical Review D: Particles, Fields, Gravitation and Cosmology, vol. 86, no. 10, Article ID 104003, 2012.

[8] J. Cortez, G. A. Mena Marugán, and J. M. Velhinho, "Quantum unitary dynamics in cosmological spacetimes," Annals of Physics, vol. 363, pp. 36-47, 2015.

[9] J. Cortez, B. Elizaga Navascués, M. Martín-Benito, G. A. Mena Marugán, and J. M. Velhinho, "Unitary evolution and uniqueness of the Fock representation of Dirac fields in cosmological spacetimes," Physical Review D: Particles, Fields, Gravitation and Cosmology, vol. 92, no. 10, 105013, 16 pages, 2015.

[10] J. Cortez, B. Elizaga Navascués, M. Martín-Benito, G. A. Mena Marugán, and J. M. Velhinho, "Unique Fock quantization of a massive fermion field in a cosmological scenario," Physical Review D: Particles, Fields, Gravitation and Cosmology, vol. 93, no. 8, Article ID 084053, 2016.

[11] J. Cortez, B. Elizaga Navascués, M. Martín-Benito, G. A. Mena Marugán, and J. M. Velhinho, "Dirac fields in flat FLRW cosmology: Uniqueness of the Fock quantization," Annals of Physics, vol. 376, pp. 76-88, 2017.

[12] J. Cortez, B. Elizaga Navascués, M. Mart n-Benito, G. A. Mena Marugán, and J. M. Velhinho, "Uniqueness of the Fock quantization of Dirac fields in $2+1$ dimensions," Physical Review D, vol. 96, no. 2, Article ID 025024, 2017.

[13] R. Verch, "Local definiteness, primarity and quasiequivalence of quasifree Hadamard quantum states in curved spacetime," Communications in Mathematical Physics, vol. 160, no. 3, pp. 507-536, 1994.

[14] C. D’Antoni and S. Hollands, "Nuclearity, local quasiequivalence and split property for Dirac quantum fields in curved spacetime," Communications in Mathematical Physics, vol. 261, no. 1, pp. 133-159, 2006.

[15] D. Shale, "Linear symmetries of free boson fields," Transactions of the American Mathematical Society, vol. 103, pp. 149-167, 1962.

[16] J. Dereziński, "Introduction to Representations of the Canonical Commutation and Anticommutation Relations," in Large Coulomb Systems, J. Dereziński and H. Siedentop, Eds., vol. 695 of Lecture Notes in Physics, Springer Berlin Heidelberg, 2006.

[17] A. N. Kolmogorov and S. V. Fomin, Elements of the Theory of Functions and Functional Analysis, Dover, England, 1999.

[18] S. Hollands, "The Hadamard condition for Dirac fields and adiabatic states on Robertson-Walker spacetimes," Communications in Mathematical Physics, vol. 216, no. 3, pp. 635-661, 2001.

[19] W. Junker and E. Schrohe, "Adiabatic vacuum states on general spacetime manifolds: definition, construction, and physical properties," A Journal of Theoretical and Mathematical Physics, vol. 3, no. 6, pp. 1113-1181, 2002.
[20] C. Lüders and J. E. Roberts, "Local quasiequivalence and adiabatic vacuum states," Communications in Mathematical Physics, vol. 134, no. 1, pp. 29-63, 1990.

[21] W. Junker, "Hadamard states, adiabatic vacua and the construction of physical states for scalar quantum fields on curved spacetime," Reviews in Mathematical Physics, vol. 8, no. 8, pp. 1091-1159, 1996. 


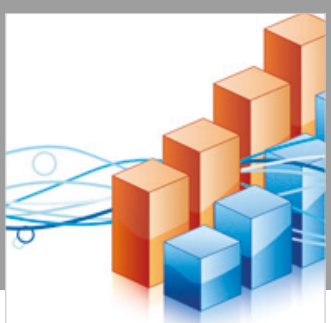

Advances in

Operations Research

\section{-n-m}
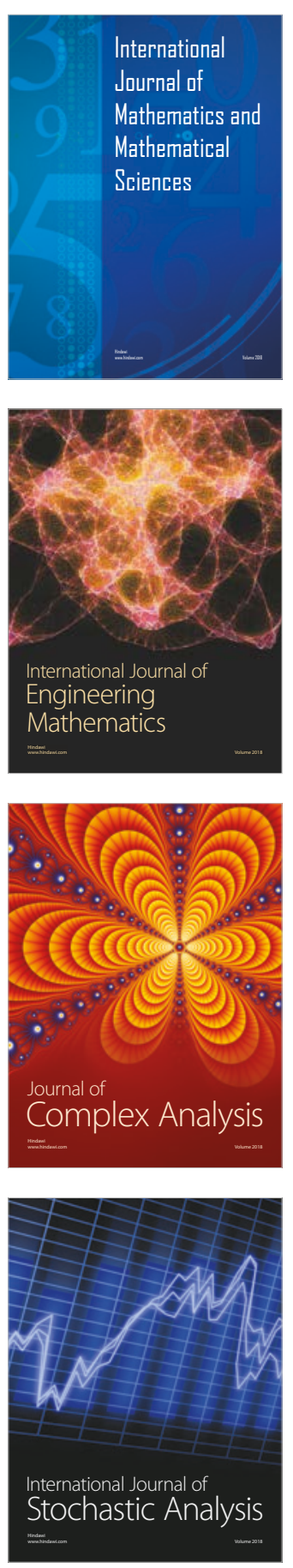
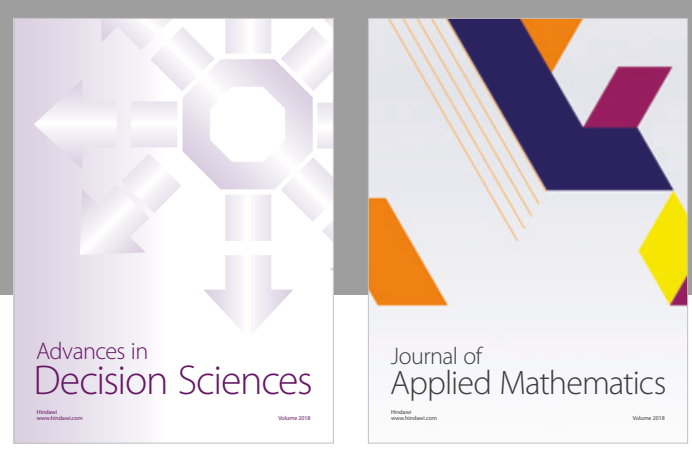

Journal of

Applied Mathematics
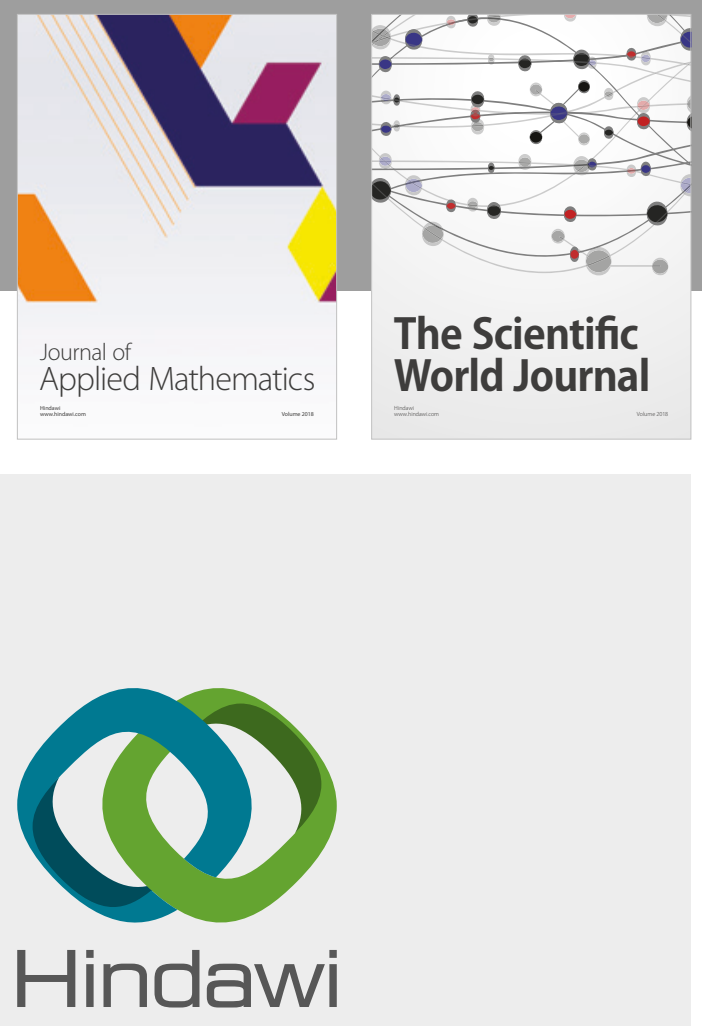

Submit your manuscripts at

www.hindawi.com

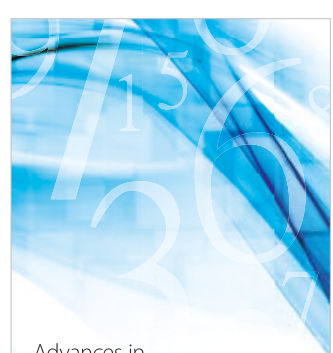

Advances in
Numerical Analysis
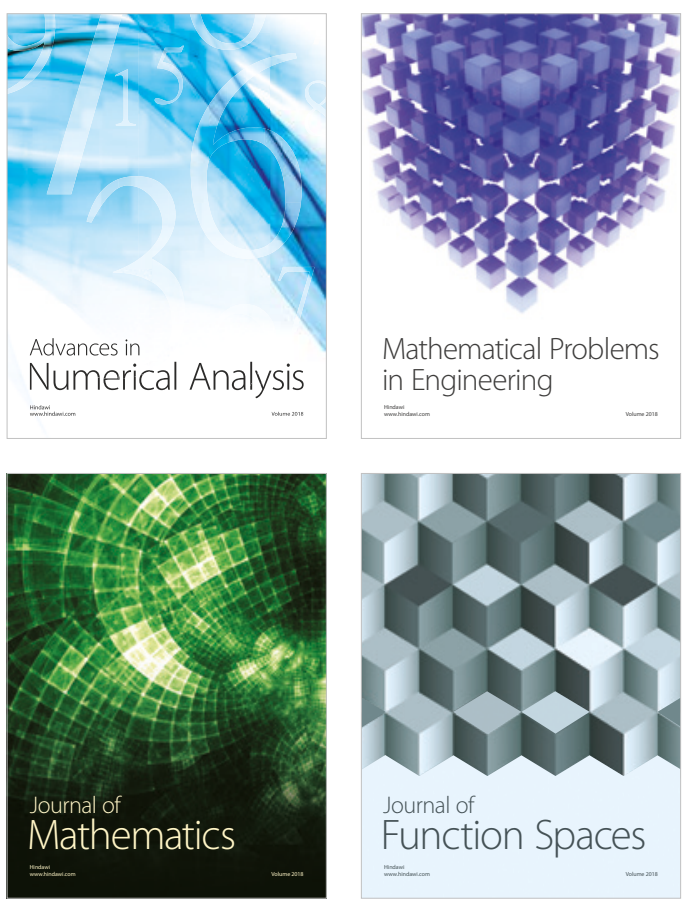

Mathematical Problems in Engineering

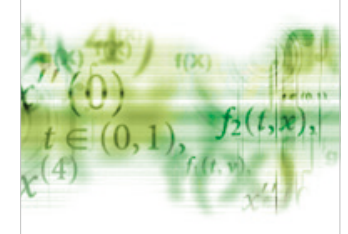

International Journal of

Differential Equations

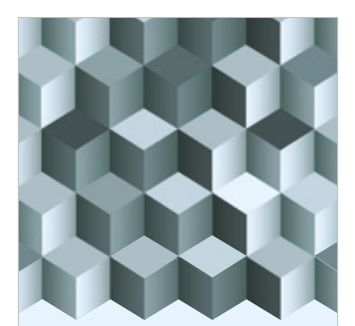

Journal of

Function Spaces

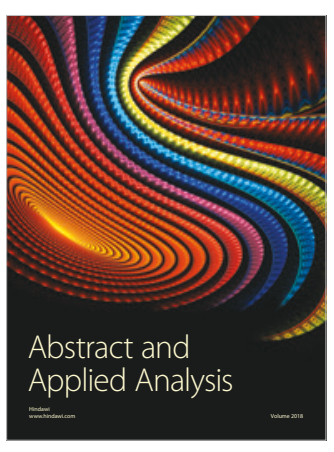

The Scientific

World Journal

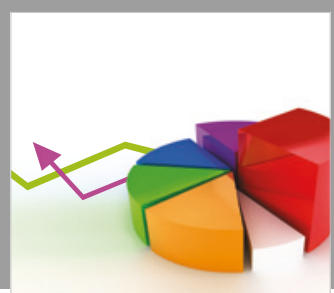

Journal of

Probability and Statistics
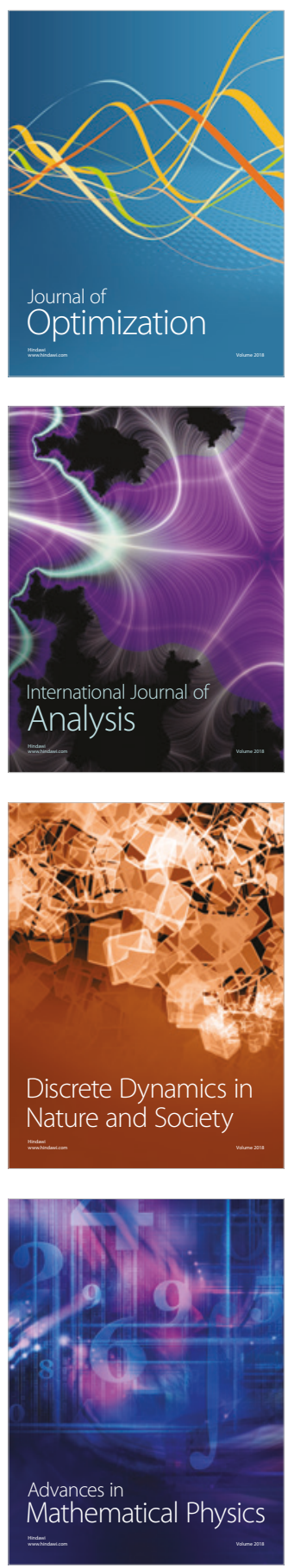